\title{
Silicon-Micromachined Gas Chromatographic Columns for the Development of Portable Detection Device
}

\author{
Jean-Baptiste Sanchez, Aline Schmitt, Franck Berger, and Christophe Mavon \\ Laboratoire de Chimie Physique et Rayonnements Alain Chambaudet, UMR CEA E4, UFR Sciences et Techniques, \\ 16 route de Gray, 25000 Besancon, France \\ Correspondence should be addressed to Jean-Baptiste Sanchez, jbsanche@univ-fcomte.fr
}

Received 2 September 2010; Accepted 5 October 2010

Academic Editor: K. Kalantar-Zadeh

Copyright ( 92010 Jean-Baptiste Sanchez et al. This is an open access article distributed under the Creative Commons Attribution License, which permits unrestricted use, distribution, and reproduction in any medium, provided the original work is properly cited.

\begin{abstract}
We report the fabrication of a gas chromatographic column module integrated on a silicon substrate and usable as a portable measurement device dedicated to the selective detection of various chemical compounds (gas or vapour). PDMS, PEG, and F13TEOS stationary phases have been prepared in order to coat the inside walls of microchannels. The microcolumn tests were performed with a mixture of hydrocarbons and ketone. After having evaluated the effectiveness of such a separation module, we showed an application by coupling a GC microcolumn with a metal oxide-based gas sensor. The best results were obtained at a low isothermal temperature mode of the GC micro-column (near the ambient temperature). The coupling between the GC microcolumn and a metal oxide gas sensor enables to obtain a rapid, reliable, and selective analysis of various chemical compounds.
\end{abstract}

\section{Introduction}

As part of human health protection and environment, the level of pollutants in the air must be as low as possible. Thus, the use of efficient systems is recommended to control the presence of contaminants in the air. At the present time, some common analytical laboratory equipments, such as gas chromatography devices (GC, GC/MS) can detect successfully different toxic gases at the ppb level [1-5]. However, their high cost and heavy technical maintenance make them impractical for on-line monitoring of pollutants. Recently, the increasing needs for inexpensive and small monitoring devices have suggested to miniaturize the analysis systems. It is well known that miniaturization offers functional and economical benefits such as a reduction of the sample size, a decrease in reagent consumption, and an inexpensive mass production [6].

The selective detection of some chemical compounds in a mixture is impossible by using a single sensor. A chromatographic separation stage is necessary to obtain a selective detection of various chemical compounds in a sample mixture. Since the initial development of gas microchromatographs by Terry in 1979 [7], different studies have been carried out to develop silicon microfabricated chromatographic columns. A not exhaustive list is given in the references section of this paper [7-13]. However, so far, all these products have remained confined in the research laboratories and are not yet commercially available. The main reason is the difficulty to produce reliable and reproducible systems. Nevertheless, the application fields of such microfluidic systems are very interesting. In particular, we can take the example of the development of selective portable gas sensors for the monitoring of the atmospheric pollution.

In this context, the general idea of this research work is to develop a separation module integrated on a silicon substrate which can be used in front of various miniaturizable sensors such as chemical gas sensor and mass spectrometer.

The aim of this work is to propose a reliable and easier procedure, first to manufacture micro-channels on a silicon substrate, then to deposit inside the micro-channels a thin film of stationary phase. To separate a wide range of chemical compounds, the solution is to prepare different stationary phases presenting various physicochemical properties (polarity, chemical inertia). 


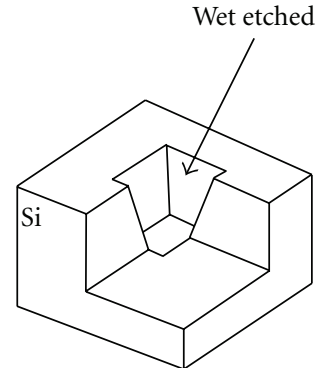

Definition of the opening access:

Position for a gas inlet tube

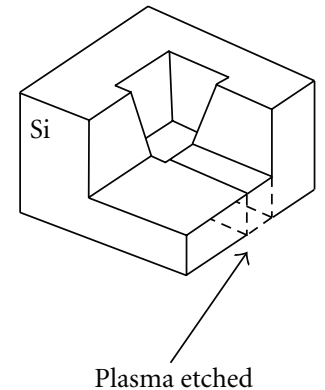

2

Micromachining of the channel

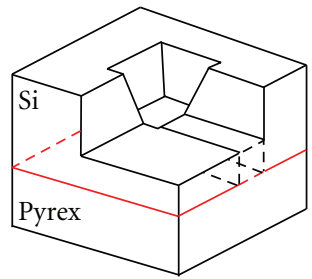

3

Anodic bonding of a pyrex wafer: definition of the three-dimensional structure

FIgURE 1: Steps involved during the manufacture of the GC micro-column.

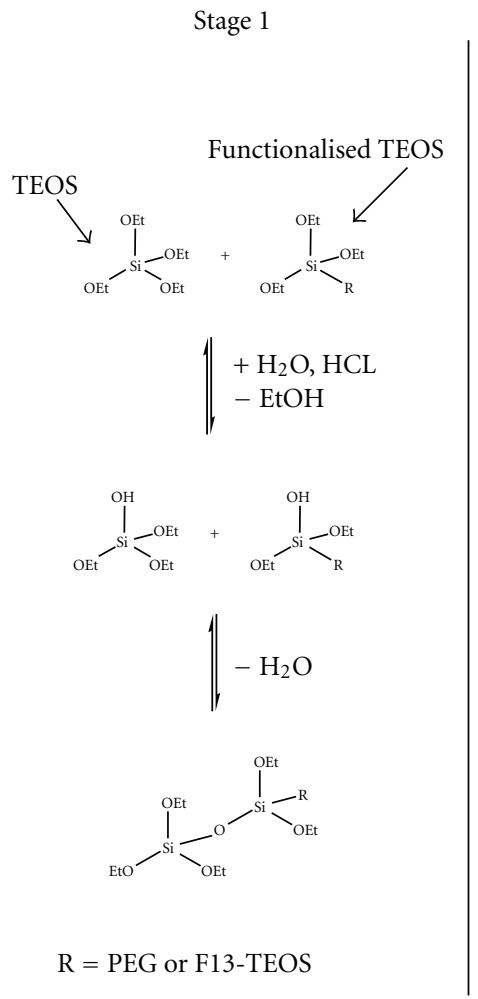

Stage 2
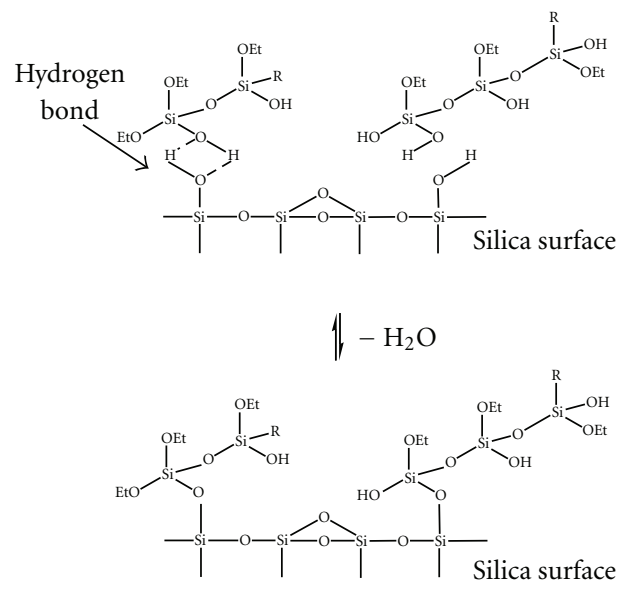

FIGURE 2: Different steps for the formation and deposition of the stationary phase using the sol-gel process.

In this paper, first we describe the complete process to realize GC microcolumns on a silicon substrate. Secondly we provide the characterization of these devices by using a mass spectrometer in order to evaluate the effectiveness of the separation. Then, we show an application of such GC microcolumns when they are used in front of a metal oxide gas sensor to obtain a microdevice for the monitoring of the atmospheric pollution.

\section{Experimental}

2.1. Fabrication of the Micro-Channels. The general geometry selected for these microstructures is a circular spiral configured like two interlocking structures in order to reduce the dimensions on the silicon wafer. This geometrical configuration makes it possible to dispose 4 GC microcolumns on a 4-inch-silicon wafer. At the beginning, the structures were 


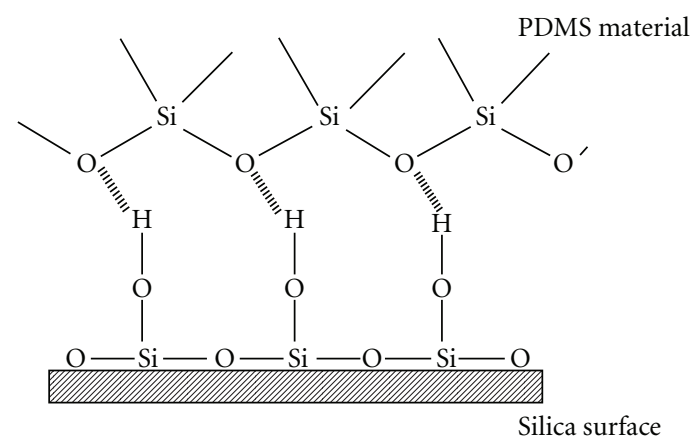

FIGURE 3: Bonding between PDMS and silica surface [16].

patterned by using standard photolithographic processes. We have used two approaches for a complete etching of the micro-channels through a silicon wafer (100).

The first etching process used a $\mathrm{KOH}$ solution to create an opening access of the micro-channels. The second fabrication stage used plasma in order to form deep narrow channels with vertical sidewalls (DRIE method). The results of the silicon wafer etching process are shown schematically in Figure 1 (steps 1 and 2).

After these two first steps, silicon substrates were coated with $\mathrm{SiO}_{2}$ by thermal oxidation $(100 \mathrm{~nm})$. The relevance is to favour the continuation of the process in order to deposit the stationary phase on the internal walls of the micro-column. A closed channel is produced by anodically bonding a Pyrex wafer to the side of the channel (Figure 1, step 3).

2.2. Stationary Phases Coating. To complete the realization of the silicon-micromachined gas chromatographic column, a stationary phase should be introduced and put into the micro-channel. For this study, we selected three stationary phases. A nonpolar stationary phase (Polydimethylsiloxane (PDMS)), a semipolar stationary phase (Polyethylene glycol (PEG)), and a great chemical inertia stationary phase (Tridecafluoro-1,1,2,2-tetrahydrooctyl-1triethoxysilane (F13-TEOS)). The choice of the coating chemical nature used depends on the analyte of interest. Typically, polar phases strongly interact with polar analytes, causing longer elution times and better separation. Likewise, non-polar phases are best suited to non-polar analytes.

Given the very small size of the micro-structure, the easiest way to deposit the stationary phase is to introduce a liquid solution and then obtain a polymerization inside the microchannel. In this case, for the preparation of PEG and F13-TEOS stationary phases, we use the so-called solgel technique for the preparation and the grafting of the stationary phase $[14,15]$.

The main interest of this process is that it makes possible to insert the still liquid solution in the micro-channel, where it reacts with the solution on the internal walls of the microcolumn to form the desired thin film of the stationary phase.
In particular, the chemical formulation of the stationary phase has been obtained by mixing tetraethoxysilane (TEOS) with PEG or F13-TEOS. The first precursor (TEOS) enabled the formation of a silica matrix grafted onto the internal walls of the GC micro-column. The two other precursors (F13TEOS or PEG) were used particularly to functionalize the whole stationary phase. Figure 2 indicates the different steps during the sol-gel process.

The anchorage to the micro-column inside walls is possible through condensation reactions between the silanol groups of the microchannel $\left(\mathrm{SiO}_{2}\right)$ and the residual silanol groups of the gel (silica matrix).

For the PDMS stationary phase, we did not use the solgel process, but we prepared the PDMS material by using a commercially available product. The PDMS used is Sylgard 184 from Dow Corning, a two-part heat curable system that is mixed with the included curing agent. Due to the high viscosity of PDMS and in order to introduce this material inside the micro-channel, we also used toluene as solvent. In this particular case, PDMS coats the silica surface through hydrogen bonds (cf. Figure 3). This is why PDMS material can be peeled from silica surface (e.g., manufacturing of microfluidic devices).

\section{Results}

3.1. Micromachined GC Column. Figure 4 shows images of the micromachined column. The column consists in a 50$\mu \mathrm{m}$-wide and $50-\mu \mathrm{m}$-deep channel with a length of about 2 meters. In order to introduce the different gases and the appropriate stationary phase into the anodically sealed column, we attached capillary tubes into holes defined by wet etching. We used special fluidic connections for chip-based analyses from Upchurch Scientific.

The micro-channels were filled with the coating solutions in order to obtain GC microcolumns. The solution reacted with the micro-channel's internal wall during 30 minutes before the circulation of a nitrogen flow which dynamically drove out the exceeded solution. The stationary phase was dried by heating the micro-channel at $50^{\circ} \mathrm{C}$ under nitrogen flow during 24 hours. Figure 5 presents various SEM micrographs of GC micro-column's cross section after the coating of the stationary phase.

For a reaction time inside the micro-structure up to 30 minutes, we notice a complete polymerization of the stationary phase (Figure 5(c)). In this case, it is impossible to circulate a carrier gas inside the micro-column. When the solution reacts less than 30 minutes inside the microchannel, we observe the presence of a thin film of the expected stationary phase(Figures 5(d) and 5(e)). The thickness is about $1 \mu \mathrm{m}$.

3.2. Microcolumn Test Results. In order to characterize the effectiveness of the three microcolumns developed in this work, we first chose to use a mass spectrometer as a chromatographic detector. Then we replaced the mass spectrometer by a chemical gas sensor. 


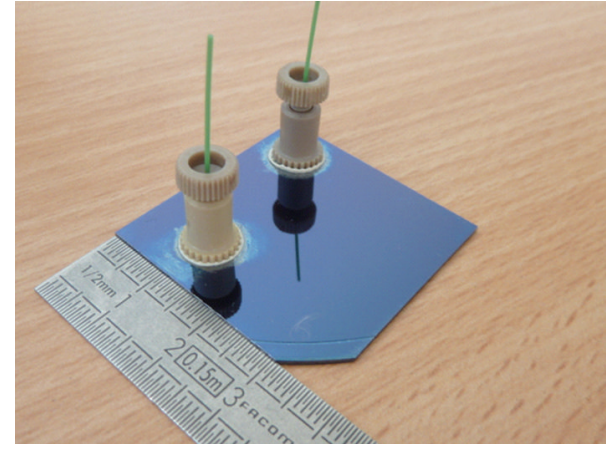

Side of the fluidic connections

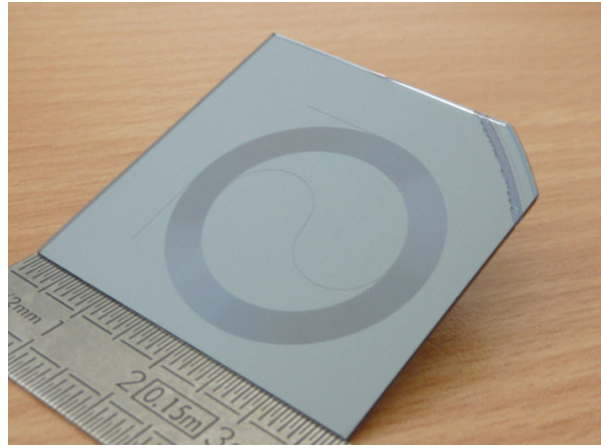

Side of the etched micro-channel

FIgURE 4: Silicon-micromachined gas chromatographic column.
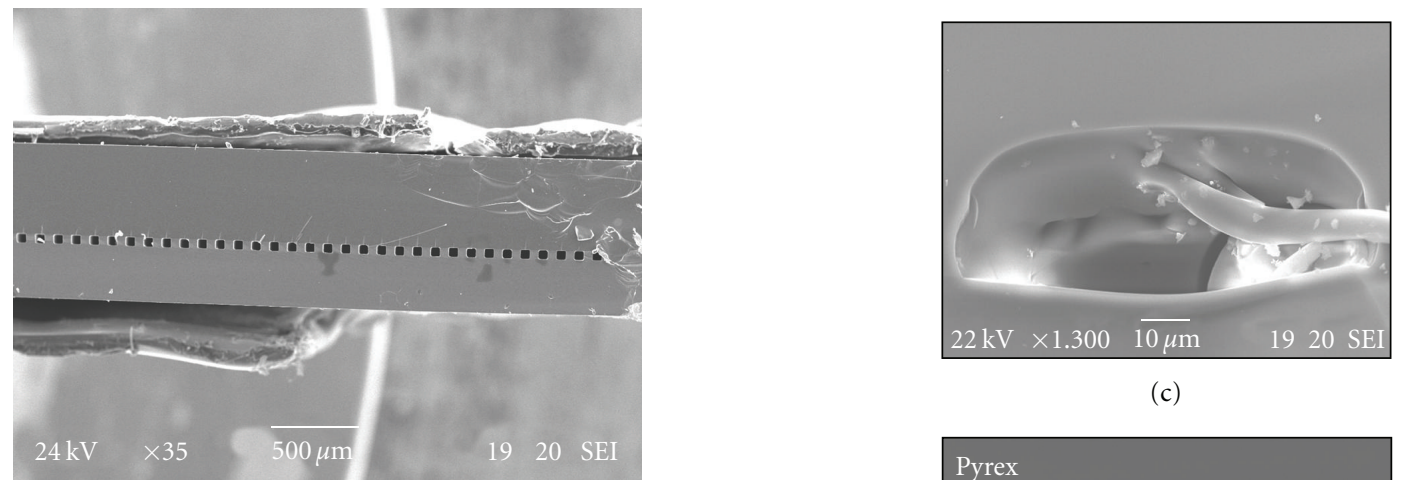

(c)

(a)

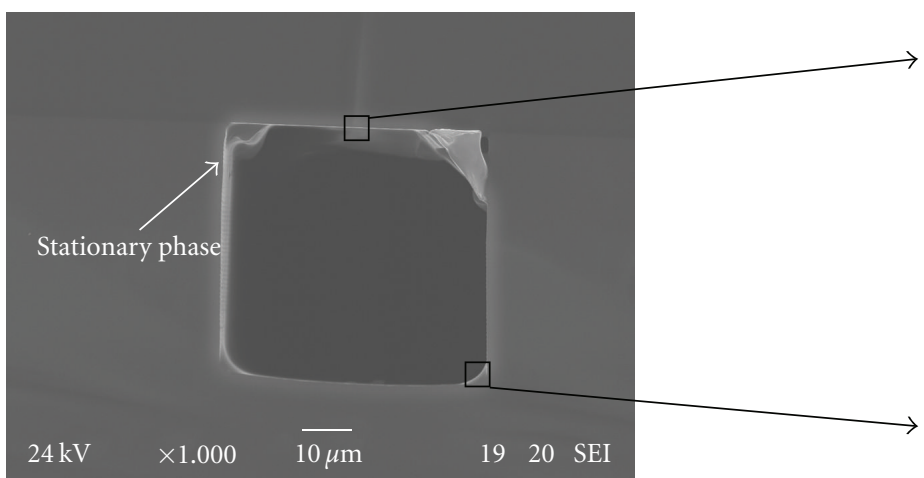

(b)

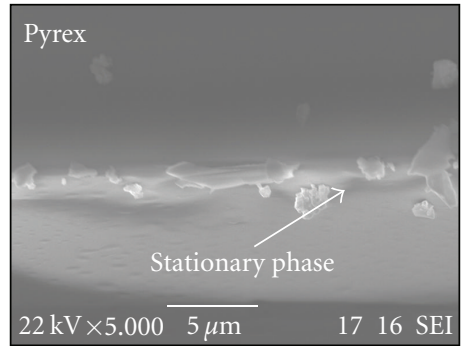

(d)

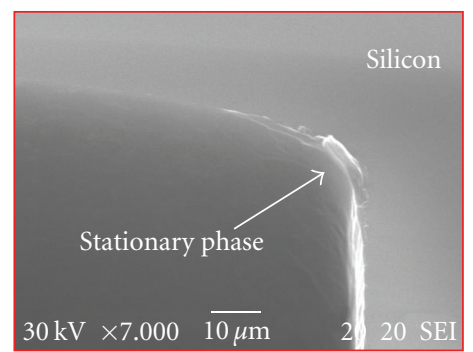

(e)

FIGURE 5: SEM micrographs of the micro-channel's cross section: (a) general view of the cross section, (b) zoon on one channel, (c) complete polymerization inside the channel, (d) thin film on the Pyrex side, and (e) thin film on the etched silicon side.

3.3. Mass Spectrometry Results. Among the various experimental factors which are necessary for the effectiveness of a chromatographic system, the following one deserves a special mention: the linear velocity of the carrier gas and the temperature of the gas chromatographic micro-column.
In our study, the linear velocity of the carrier gas depends directly on the pressure of the carrier gas applied ahead of the GC micro-column. Thus, with an increase of the inlet pressure, we notice an increase of the carrier gas linear velocity. The experimental part of this study aimed at 


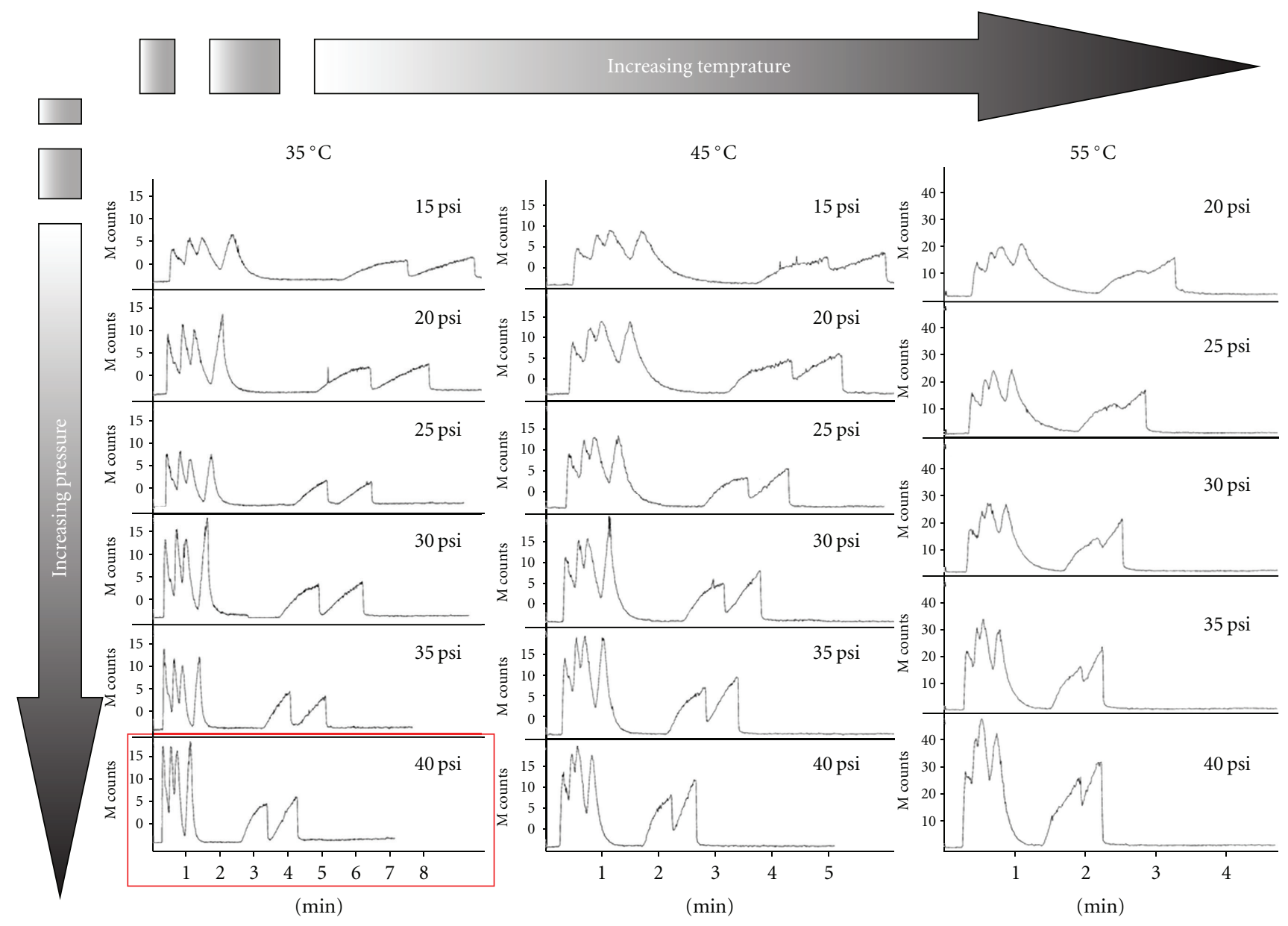

FIgURE 6: Chromatograms obtained with the GC microcolumn coated with PDMS.

highlighting the influence of these parameters to determine the optimum conditions for the analysis of a complex gas sample with a GC microcolumn.

The mixture is composed by an equal mixture of 6 chemical compounds including a ketone and various aliphatic and aromatic hydrocarbons (toluene, benzene, ortho-xylene, nonane, and heptane).

On Figure 6, we notice that the optimum parameters are a temperature of about $35^{\circ} \mathrm{C}$ (the temperature of the GC microcolumn) and an inlet gas pressure of about 40 psi. In these experimental conditions, it is possible to obtain the complete elution and separation of each chemical compound contained in the complex mixture (6 pollutants). The chromatographic peak indexation is obtained thanks to the use of the mass spectrometer. The exit order gives for this GC microcolumn: acetone, benzene, toluene, heptane, ortho-xylene, and nonane.

From these chromatograms, we also notice that less than 5 minutes are enough to identify all compounds.

Working at low analysis temperature to obtain good separations has the advantage to decrease the electrical consumption. This is a very interesting result because, most of the time, chromatographic separations can be performed either at a high isothermally temperature or with a temperature ramp, so it induces an important electrical consumption. Currently, the development of miniaturized system is strongly restricted by the electrical alimentation of the various elements.

By fixing these experimental conditions (temperature of the $\mathrm{GC}$ microcolumn equal to $35^{\circ} \mathrm{C}$, inlet pressure of about $40 \mathrm{psi}$ ), we obtained various chromatograms with the three GC microcolumns available in this study. Figure 7 shows three chromatograms corresponding to the analysis of the same mixture by the three GC microcolumns. These analyses clearly highlight the influence of the chemical nature of the stationary phase on the elution and the separation of chemical compounds through the GC microcolumn. Mass spectrometer enables to attribute each chemical compound to each chromatographic peak.

As we can see from these chromatograms, the PDMS stationary phase seems to be very suitable for the analysis of a mixture of hydrocarbons (aliphatic and aromatic). On the other side, in the same experimental conditions with the PEG stationary phase, the exit order of each chemical compound is modified, and the chromatographic peaks are not well defined. For the F13-TEOS, aliphatic hydrocarbons compounds and ketone are not eluate. Only aromatic hydrocarbons are separate. This stationary phase is 


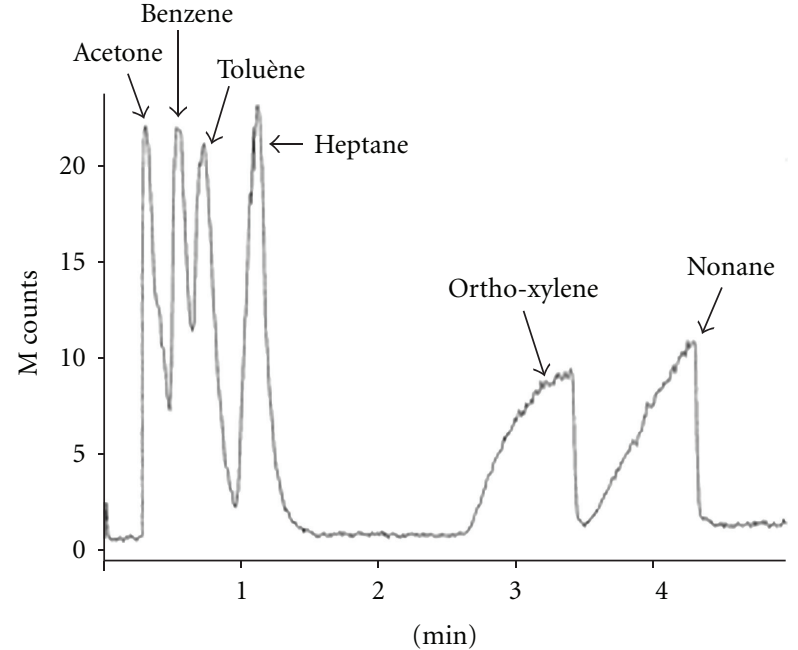

(a)

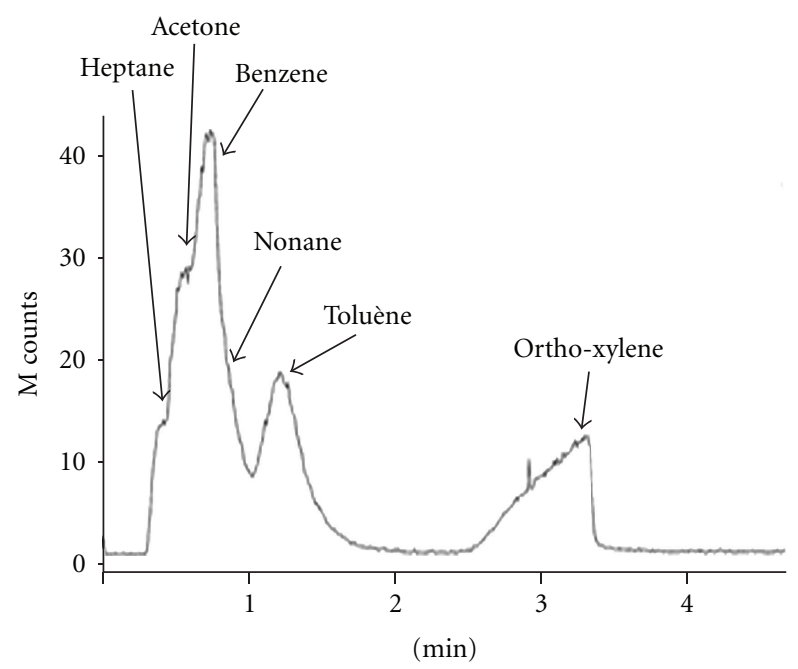

(b)

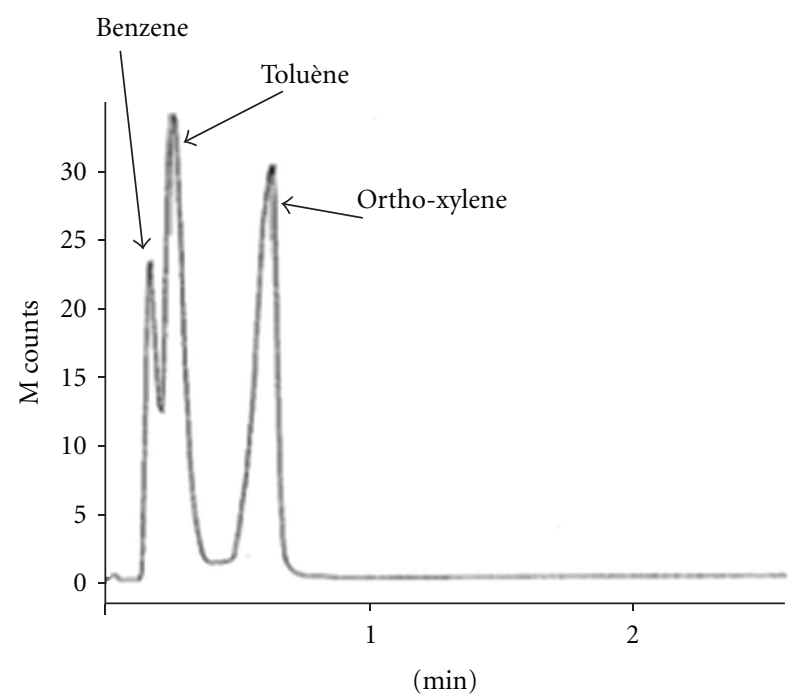

(c)

Figure 7: Chromatograms obtained with the three GC microcolumns: PDMS (a), PEG, (b) and F13-TEOS (c).

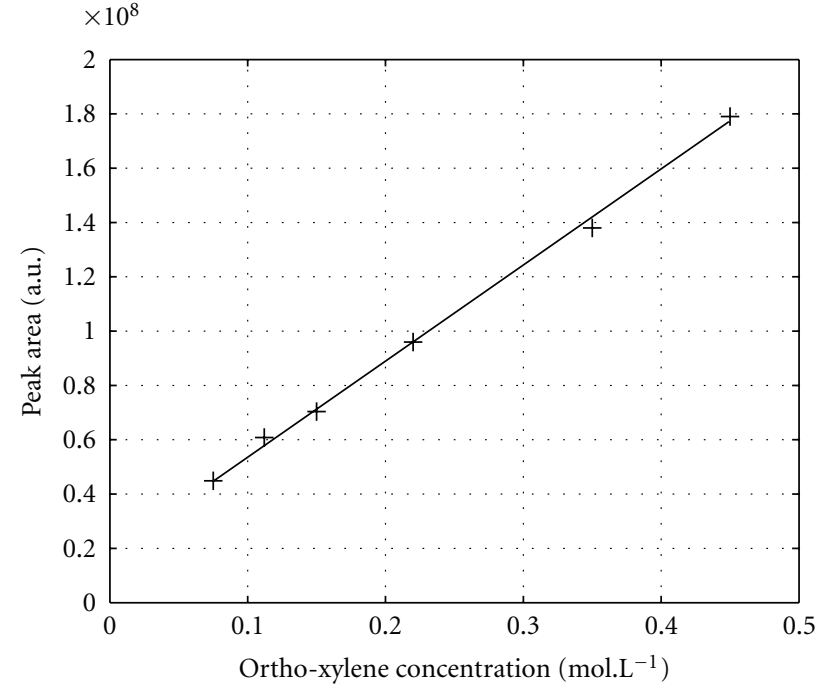

Figure 8: Calibrating curve giving the Ortho-xylene area peak versus its concentration in the mixture.

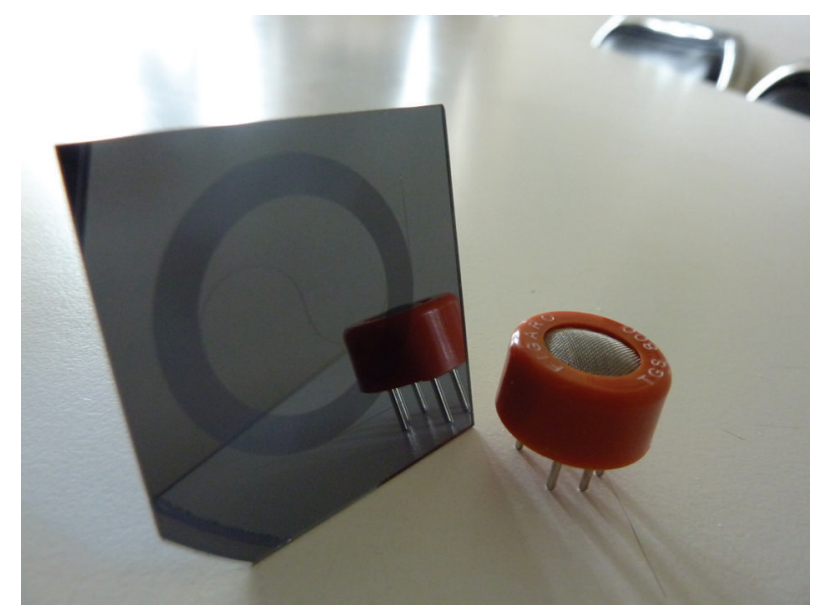

FIgURE 9: Example of the coupling between the GC micro-column and a commercially available metal oxide based gas sensor.

very interesting for the analysis of VOCs in the atmosphere. Indeed, the analysis time is really short and realized in an isothermal mode near the ambient temperature.

A quantitave analysis is also possible with this kind of GC microcolumns. For example, we quantitatively studied the progressive dilution of o-xylene in the complex mixture. By calculating the area of the o-xylene chromatographic peak, we plotted the calibrating curve (cf. Figure 8).

Figure 8 indicates a linear behavior of the calibrating curve. With this kind of representation, it is possible to evaluate the concentration of a pollutant (here ortho-xylene compound) in a mixture in a good separation condition in the GC micro-column.

3.4. Chemical Microsensor Results. The microfabricated GC columns operate efficiently under low volumetric flow rates and thus require detectors with very small residence times. 


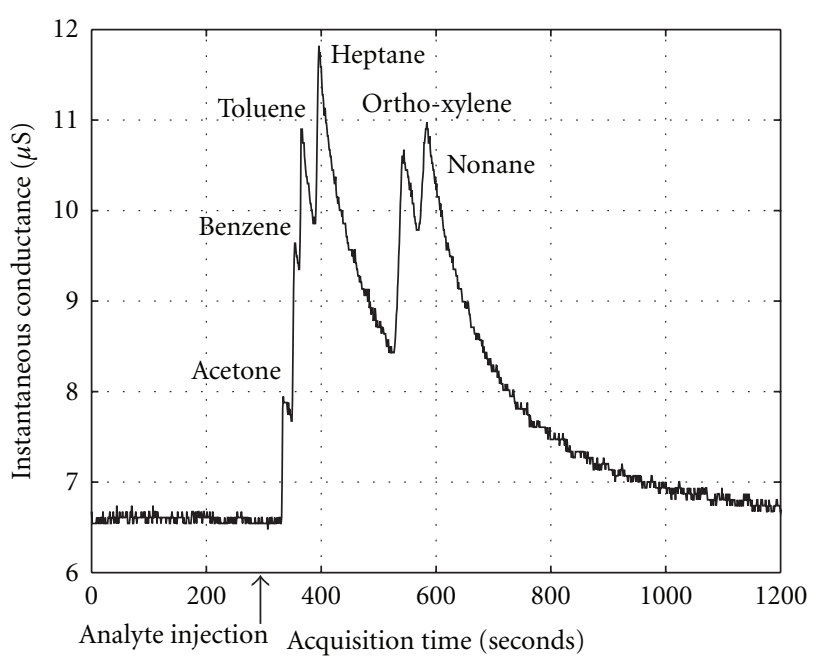

(a) PDMS GC microcolumn

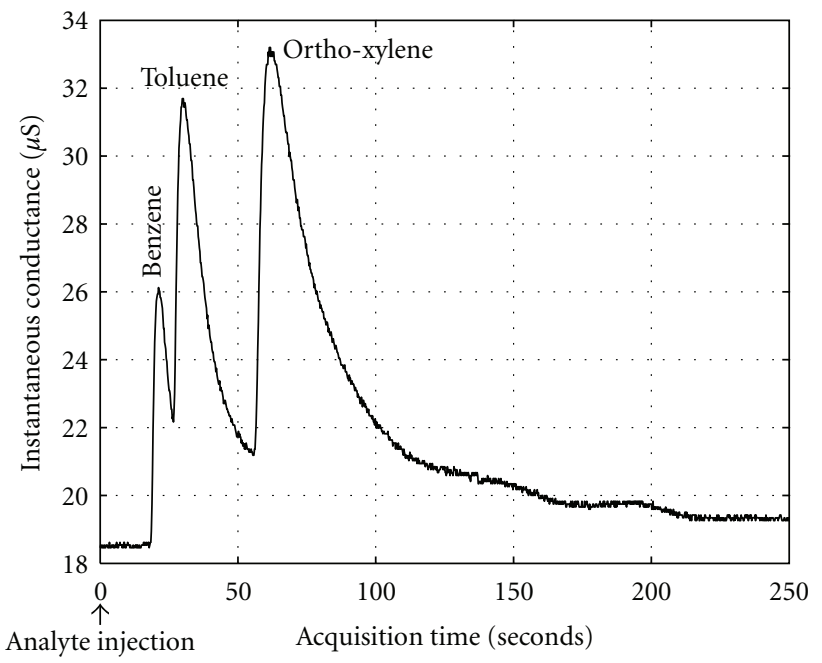

(b) F13-TEOS GC microcolumn

Figure 10: Chromatograms obtained by coupling GC microcolumns with a metal oxide-based gas sensor.

An application of these GC microcolumns is to be used in front of chemical gas sensors such as metal oxide based gas sensors (MOX gas sensors). The aim is to obtain a miniaturized and portable device, which is able to give a selective detection of various pollutants in the atmosphere. MOX gas sensors can be used as a chromatographic detector [17]. The measurement of the change in the resistive value of the metal oxide material due to the chemical reaction serves as sensing signal. The electrical conductance of the sensitive layer $\left(\mathrm{SnO}_{2}\right)$ corresponds both with the gas concentration and with the physicochemical properties of the detected gas [18]. The effect of gases on the sensor's electrical properties is recorded by monitoring the variations in the sensor's instantaneous conductance versus time, for a temperature of the sensitive element $\left(\mathrm{SnO}_{2}\right)$ fixed at $773 \mathrm{~K}$.

Figure 9 shows a picture of a commercially available metal oxide gas sensor (TGS gas sensor from Figaro) in front of a GC micro-column realized in this study.
Figure 10 represents two chromatograms obtained by coupling a metal oxide-based gas sensor with GC microcolumns. The tests were performed by using PDMS and F13TEOS stationary phases.

The exit of each chemical compound and its interaction with the sensitive element of the gas sensor can be identified thanks to the ascending part of each chromatographic peak. Given these considerations, it is possible to discriminate each chemical compound contained in the mixture. These tests have been reproduced several times and we did not observe any shift in the analysis time and in the exit order of the chemical compounds.

Once again, in this experimental configuration, we noticed a rapid analysis of the pollutants in the mixture.

\section{Conclusion}

In this paper, our aim has been to propose a process to develop gas chromatographic microcolumns integrated on a silicon substrate for the selective analysis of various chemical compounds. The formulation of different stationary phases in this study enables to analyse a wide range of chemical compounds, in particular hydrocarbons. With these microdevices we can obtain a qualitative and quantitative analysis of the pollutants in a mixture. For each GC microcolumn developed in this research work, the best performances are obtained at a low isothermal mode (near the ambient temperature). Moreover, we noticed that these microdevices, can be positioned in front of different sensors and in particular metal oxide gas sensor. From a technological development point of view and considering the clean room facilities, it is possible to integrate GC microcolumns and MOX gas sensor on a unique substrate in order to obtain an analytical platform for the selective detection of pollutants.

\section{Acknowledgment}

The authors gratefully acknowledge the technical asistance of the MIMENTO team of Femto-ST Institute for the help during the realization of the microsystems.

\section{References}

[1] J. J. Johnston, D. A. Goldade, D. J. Kohler, and J. L. Cummings, "Determination of white phosphorus residues in ducks: an atomic emission detection/compound-independent calibration-based method of generating residue data for risk assessment and environmental monitoring," Environmental Science and Technology, vol. 34, no. 9, pp. 1856-1861, 2000.

[2] J. A. De Gouw, C. J. Howard, T. G. Custer, B. M. Baker, and R. Fall, "Proton-transfer chemical-ionization mass spectrometry allows real-time analysis of volatile organic compounds released from cutting and drying of crops," Environmental Science and Technology, vol. 34, no. 12, pp. 2640-2648, 2000.

[3] L. Charles, L. S. Riter, and R. G. Cooks, "Direct analysis of semivolatile organic compounds in air by atmospheric pressure chemical ionization mass spectrometry," Analytical Chemistry, vol. 73, no. 21, pp. 5061-5065, 2001. 
[4] B. O. Galle, J. Samuelsson, B. H. Svensson, and G. Borjesson, "Measurements of methane emissions from landfills using a time correlation tracer method based on FTIR absorption spectroscopy," Environmental Science and Technology, vol. 35, no. 1, pp. 21-25, 2001.

[5] X. Hu, J. Nicholas, J.-J. Zhang, T. M. Linjewile, P. D. Filippis, and P. K. Agarwal, "The destruction of $\mathrm{N}_{2} \mathrm{O}$ in a pulsed corona discharge reactor," Fuel, vol. 81, no. 10, pp. 1259-1268, 2002.

[6] B. van der Schoot, E. Verpoorte, S. Jeanneret, A. Manz, and N. de Rooij, "Microsystems for analysis in flowing solutions," in Proceedings 1st International Symposium on Micro Total Analysis Systems, p. 21, Twente, The Netherlands, 1994.

[7] S. C. Terry, J. H. Jerman, and J. B. Angell, "A gas chromatographic air analyzer fabricated on a silicon wafer," IEEE Transactions on Electron Devices, vol. 26, no. 12, pp. 18801886, 1979.

[8] R. R. Reston and E. S. JR. Kolesar, "Silicon-micromachined gas chromatography system used to separate and detect ammonia and nitrogen dioxide. Part I: design, fabrication, and integration of the gas chromatography system," Journal of Microelectromechanical Systems, vol. 3, no. 4, pp. 134-146, 1994.

[9] C. M. Matzke, R. J. Kottenstette, S. A. Casalnuovo et al., "Microfabricated silicon gas chromatographic microchannels: fabrication and performance," in Micromachining and Microfabrication Process Technology IV, vol. 3511 of Proceedings of SPIE, pp. 262-268, Santa Clara, Calif, USA, September 1998.

[10] Wiranto, Malcolm R. Haskard, Dennis E. Mulcahy, David E. Davey, and Ernest F. Dawes, "Microengineered open tubular columns for GC analysis," in Electronics and Structures for MEMS, vol. 3891 of Proceedings of SPIE, pp. 168-177, Queensland, Australia, 1999.

[11] G. R. Lambertus, C. S. Fix, S. M. Reidy et al., "Silicon microfabricated column with microfabricated differential mobility spectrometer for GC analysis of volatile organic compounds," Analytical Chemistry, vol. 77, no. 23, pp. 7563-7571, 2005.

[12] S.-I. Ohira and K. Toda, "Micro gas analyzers for environmental and medical applications," Analytica Chimica Acta, vol. 619, no. 2, pp. 143-156, 2008.

[13] A. D. Radadia, R. I. Masel, M. A. Shannon, J. P. Jerrell, and K. R. Cadwallader, "Micromachined GC columns for fast separation of organophosphonate and organosulfur compounds," Analytical Chemistry, vol. 80, no. 11, pp. 4087-4094, 2008.

[14] S. Constantin and R. Freitag, "Preparation of stationary phases for open-tubular capillary electrochromatography using the sol-gel method," Journal of Chromatography A, vol. 887, no. 1-2, pp. 253-263, 2000.

[15] C. J. Brinker, "Hydrolysis and condensation of silicates: effects on structure," Journal of Non-Crystalline Solids, vol. 100, no. 1-3, pp. 31-50, 1988.

[16] J.-N. Paquien, Etude des propriétés rhéologiques et de l'état de dispersion de suspensions PDMS/silice, thesis, Ecole doctorale des matériaux de Lyon, 2003, 03ISAL 0067.

[17] J.-B. Sanchez, F. Berger, M. Fromm, and M.-H. Nadal, "Use of a chromatographic column to improve the selectivity of the $\mathrm{SnO}_{2}$ gas sensors: first approach towards a miniaturised device and selective with hydrogen fluoride vapours," Sensors and Actuators B, vol. 106, no. 2, pp. 823-831, 2005.

[18] L. Gajdošík, "The concentration measurement with $\mathrm{SnO}_{2}$ gas sensor operated in the dynamic regime," Sensors and Actuators $B$, vol. 106, no. 2, pp. 691-699, 2005. 

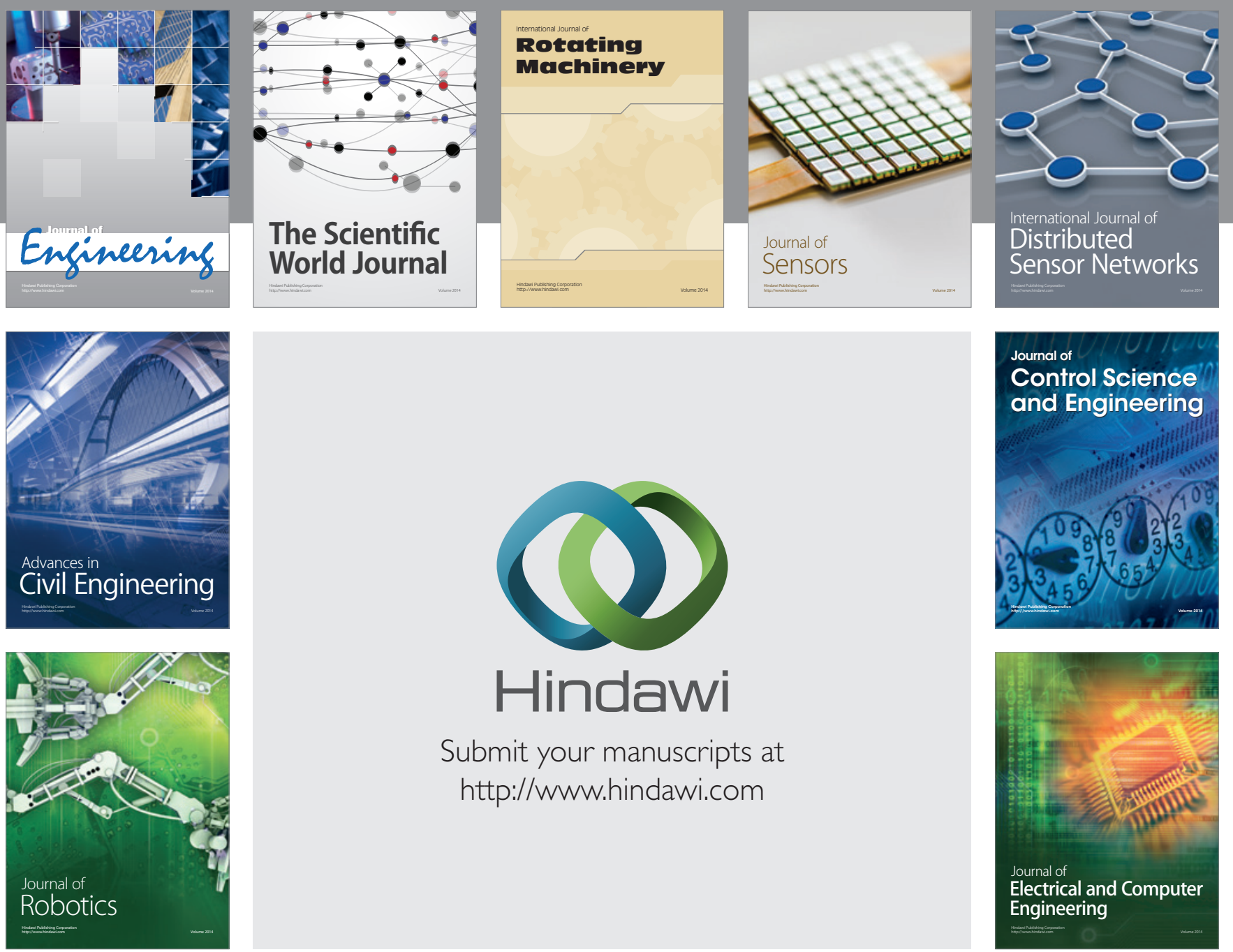

Submit your manuscripts at

http://www.hindawi.com
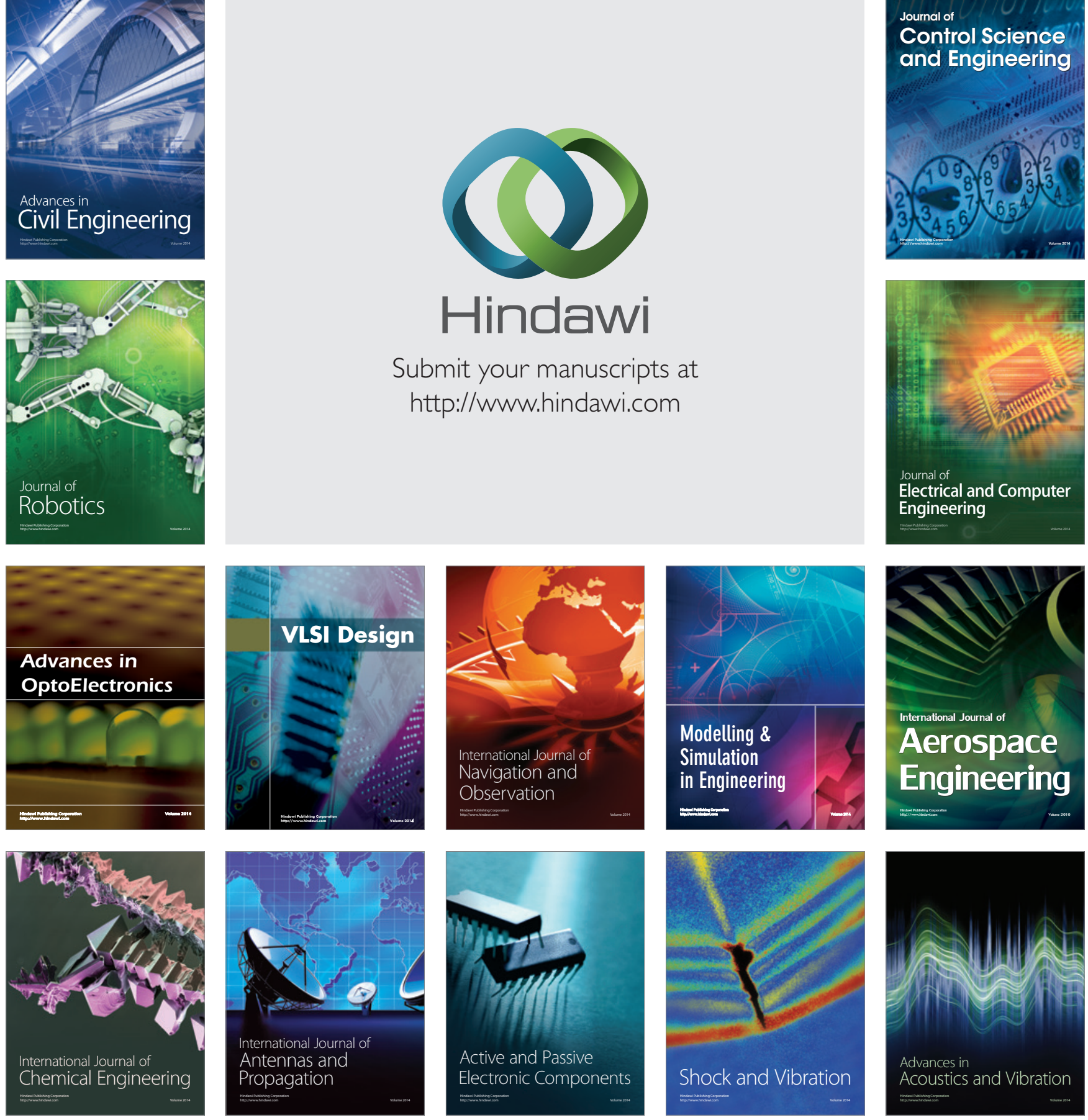